\title{
Development of Ultra-fast 3D Silicon Detectors: 3D Simulation and Modeling of 3D-trench Electrode Detector
}

\author{
Man-Wen $\mathrm{LIU}^{1,2, \mathrm{a}}$ and Zheng $\mathrm{LI}^{1,2, \mathrm{~b},{ }^{*}}$ \\ ${ }^{1}$ Engineering laboratory of Special Functional thin film Materials, School (Faculty) of Materials \\ Science and Engineering, Xiangtan University, Xiangtan 411105, China. \\ ${ }^{2}$ Center for semiconductor Particle and photon Imaging Detector Development and Fabrication, \\ Xiangtan University, Xiangtan 411105, China. \\ e-mail: amwliu1993@163.com, blizheng@xtu.edu.cn
}

Keywords: ultra-fast 3D silicon detector; electric potential; electric field; response time; breakdown consideration

\begin{abstract}
A square 3D-trench electrode detector structure based on the fast readout electronics is simulated in this paper using TCAD tools. Due to the small size of this structure, the detector is intrinsically rad-hard and it's response time can be as fast as 10's of ps. Electric characteristics including electric potential and electric field have been simulated. From those characteristics, we focus on studying the improvement of the detector compared to all column electrode ultra-fast 3D silicon detector in electric potential and electric field distributions, etc. For example, the "slow region" in the center of all column electrode detector will not appear in our new detector structure. Furthermore, we take the breakdown consideration of the 3D-trench electrode detector on this paper.
\end{abstract}

\section{Introduction}

With the relatively fast readout electronics was available in real use [1], the silicon radiation detector with particularly fast velocity and response time had been introduced. For instance, fast silicon detectors have been used in the pioneering UA2 experiment at CERN, with the width of the shaped signal is $2 \mu \mathrm{s}$ at half amplitude and $4 \mu \mathrm{s}$ at the base [2]. The detector array used at colliders which have short inter-collision times will require a further increase in speed. A current amplifier with a rise-time of $4 \mathrm{~ns}$ and a pulse width at the base of $30 \mathrm{~ns}$ was developed to read out fast planar detectors [3]. A silicon detector system allowing higher speeds in timing using were developed. Silicon detectors with 3D $\mathrm{n}+$ and $\mathrm{p}+$ doped column electrodes that penetrate through the silicon bulk [4-5] have been developed, in which charge from long tracks to be collected in a rapid, smooth high current burst, as shown in Figure 1. Also, fabrication technology of making 3D silicon detectors with increased speed, sub-nanosecond time resolution fast current amplifiers, and constant-fraction comparators or fast wave-form recorders have been studied [6]. Developments in integrated circuit technology fabrication permit the design and fabrication of even higher speed current amplifiers [7-8]. Column electrodes are usually formed by etching holes with a diameter in the order of $10 \mathrm{~m}$ and diffusing dopant gasses into the surrounding single-crystal silicon. 


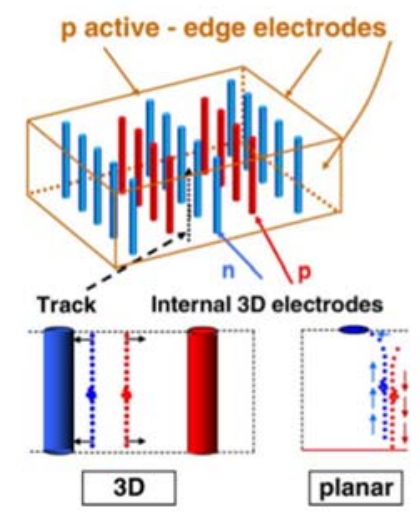

Fig.1 Schematic diagram of 3D sensor with normally-incident track.

One possible use of the fast timing resolution may be in small-angle, far-forward detectors at colliders where relative timing of the two scattered particles could locate their vertex position among several possible vertices [6]. In 2010, Zheng Li proposed a new structure with a column electrode enclosed by a trench electrode (3D-Trench Electrode Detectors), which eliminates the electric potential saddle point existed in the all column electrode detectors, and has a better electrical field distribution [9]. Later, more works focused on the radiation hard characteristics of the new structure have been performed [10-13]. From these works, we can get the conclusion that this kind of structures are radiation hard and can be used in harsh radiation environment such as HL-LHC. However, no studies have been carried out on them in terms of using them as ultra fast detectors.

In this article, we further reduce the distance of the electrodes in a square 3D-trench electrode structure of detector cell to make the responding time to pico-second level, as shown in Fig.2. In order to get fast charge collection time down to 10's of ps, we further reduce the electrode spacing to $10 \mu \mathrm{m}$ and even $5 \mu \mathrm{m}$. The detector thickness is chosen as $200 \mu \mathrm{m}$. With improved processing technology at Fondazione Bruno Kessler (FBK), Trento, Italy, columns and trench with $5 \mu \mathrm{m}$ width can be etched up to $200 \mu \mathrm{m}$ using their Deep Reactive Ion Etching system with aspect ratio up to 30:1 [14].

\section{D Simulation and Modeling of the Novel Structure}

Shown in Fig. 2 is a single cell of the detector simulated in this work. Depths of the p+ trench electrode and the $\mathrm{n}+$ column electrode are $200 \mu \mathrm{m}$, extending into the $200 \mu \mathrm{m}$ thick n-type Si bulk. In this article, we call the enclosed-trench electrode as 3D-trench electrode. Here just for the purpose of clear demonstration we chose the etch-through shell-electrode in simulation. In real fabrication, however, one either needs a support wafer or does not etch the shell-electrode all the way through. The $\mathrm{n}+$ column electrode and the $\mathrm{p}+3 \mathrm{D}$-trench electrode are covered by aluminum layers on the detector surface, while the area between electrodes on the detector top side is covered by a silicon oxide layer. The entire bottom side of detector is uniformly covered by a 1 micron thick silicon oxide layer. The distance between the electrodes is $5 \mu \mathrm{m}$ or $10 \mu \mathrm{m}$. The width of the trench is $5 \mu \mathrm{m}$, which can be realized with today's new etching technology on wafers with relatively thin thickness ( $\leqq 200$ $\mu \mathrm{m}$ ). The doping concentrations of the column and 3D-trench electrodes are 1x1019 cm-3. (boron for $\mathrm{p}+3 \mathrm{D}$-trench electrode and phosphorous for $\mathrm{n}+$ column electrode, respectively). For a detector with spacing of $10 \mu \mathrm{m}$, collection electrodes can be read out using a bump bonding technology of $10 \mu \mathrm{m}$ bumps. Although technically bump bonding technology of $10 \mu \mathrm{m}$ bumps may not be used for a detector with spacing of $5 \mu \mathrm{m}$, with future improvements of bump bonding technology, it may also be realized. We therefore went ahead to simulate this extreme situation for future applications. 


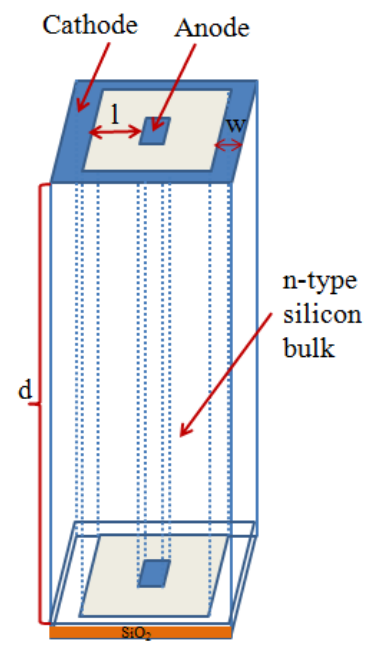

Fig.2 3D device structure in square shape with 3D-trench electrode.

Fig. 3 is the top view (although it looks up) of a square 3D-trench electrode detector with an electrode spacing of $5 \mu \mathrm{m}$ a simulation-generated structure plot using Silvaco's 3D TCAD tool [15]. The aim of the numerical simulations is to investigate the minimum bias voltages needed for carriers, generated by incident particles/photons, to reach the saturation drift velocity in the most part of the detector volume. Detector depletion voltages will also be determined.
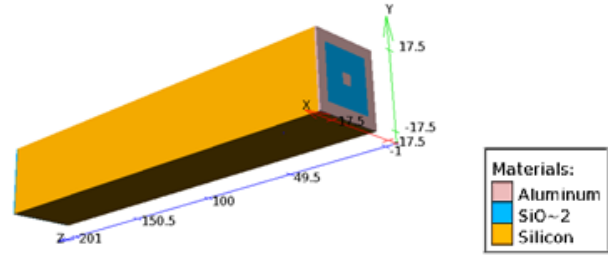

Fig.3 Detector structure simulated in this work with $10 \mu \mathrm{m}$ electrode spacing.

\section{Electrical Characteristics}

Fig. 4 shows electric potential profiles of two structures with different electrode spacings. We put negative voltages on cathode electrodes and zero bias on anode electrodes. Bias voltages are -8 volts for the left one in Fig. 4 and -4 volts for the right one in Fig. 4 . As one can see in Fig. 4 that values of electric potential at $p+$ electrodes are about those of the applied bias voltages ( -8 volts and -4 volts in our case), and those at $\mathrm{n}+$ electrodes are 0 volt. The potential at the bulk of the silicon is uniform distributed. These potential distributions clearly overcome the saddle shape potential of traditional column detector where a zero electric field exists near the detector geometry center regardless of what value the bias voltage is.

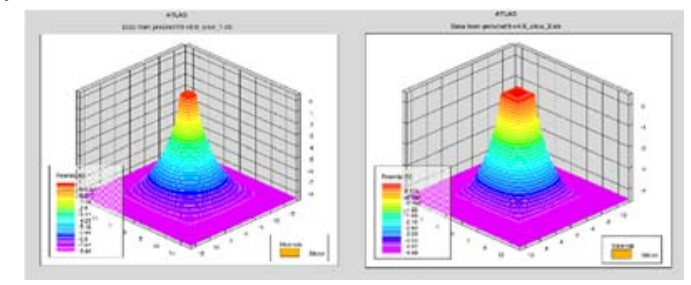

Fig.4 Electric potential of detector with an electrode spacing of $10 \mu \mathrm{m}$ and $5 \mu \mathrm{m}$.

The bias voltage are -8 volts and -4 volts.

Now, we estimate the minimum bias voltage needed to reach the carrier saturation drift velocity in the detector. Carrier (electrons here) drift velocity can be written as: 


$$
v_{d r}=\frac{\mu_{e} E}{1+\frac{\mu_{e} E}{v_{s}}}
$$

Where vdr is the drift velocity of electrons induced by the incident particle, vs is the saturation velocity for electrons, and $\mathrm{E}$ is the electric field in the detector.

To reach saturation velocity, $V_{d r}=V_{s}=1 \times 10^{7} \mathrm{~cm} / \mathrm{s}$, we must have $E>V_{s} / \mu_{e}$, from $\mu_{e}=1450 \mathrm{~cm}^{2} / \mathrm{V} / \mathrm{s}$, we can get $E \gg 10^{4} \mathrm{~V} / \mathrm{cm}$. So we chose the minimum electric field to reach the carrier saturation drift velocity as $10^{4} \mathrm{~V} / \mathrm{cm}$. When the carrier saturation drift velocity is reached, the charge collection time is then $t_{c}=L_{p} / V_{s}$. The relationship between the average electric field $\mathrm{E}$ and the bias voltage $\mathrm{V}$ can be written as: $E=V / L_{P}$, then we have:

$$
\frac{V_{\min }}{L_{p}}=\frac{V_{s}}{\mu_{e}}
$$

where Vmin is the minimum voltage needed for carriers to reach the carrier saturation drift velocity:

$$
V_{\min }=\frac{V_{s}}{\mu_{e}} \times L_{p}
$$

For example, for $\mathrm{Lp}=5 \mu \mathrm{m}, \mathrm{Vmin}$ is about 4 volts, and the charge collection time is about 50 ps. Similarly, for $L p=10 \mu \mathrm{m}, \mathrm{Vmin}$ is about 8 volts. The charge collection time is about $100 \mathrm{ps.} \mathrm{While}$ for $\mathrm{Lp}=20 \mu \mathrm{m}$, Vmin is 16 volts, and the charge collection time is about $200 \mathrm{ps}$, etc.

In the full 3D simulations of this work, we carried out simulations around these estimated minimum voltages, and use the electric field value of $104 \mathrm{~V} / \mathrm{cm}$ to determine if most of the detector volume reaches the carrier saturation drift velocity condition. On the other hand, our full 3D simulations of the detector can verify what we calculated above. Profiles of simulated detector electric field will be shown below.

From simulations of the electric field profiles, as shown from Fig. 5, we observe that in the majority regions of detectors, the electric field already reaches to $104 \mathrm{~V} / \mathrm{cm}$ when the absolute value of bias voltage $\mathrm{V}$ is larger 8 volts for $\mathrm{Lp}=10 \mu \mathrm{m}$, respectively. Fig. 5 is simulated electric field profiles at bias voltages for a detector with electrode spacing of $10 \mu \mathrm{m}(\mathrm{Lp}=10 \mu \mathrm{m})$. As shown in Fig.5, the electric field reaches $104 \mathrm{~V} / \mathrm{cm}$ in most regions between $\mathrm{p}+$ and $\mathrm{n}+$ electrodes of the detector when the absolute value of bias voltage is larger than 8 volts, which ensures the carrier saturation drift velocity condition, and therefore a fast charge collection time of about $100 \mathrm{ps}$. When $\mathrm{Lp}$ is $5 \mu \mathrm{m}$ and bias voltage $\mathrm{V}$ is -6 volts as we calculated above, we can therefore get a minimum carrier drift time of about $50 \mathrm{ps}$ in these regions of the detector at a bias voltage of $|\mathrm{V}| \geq 4 \mathrm{~V}$ for Lp $=5 \mu \mathrm{m}$. 


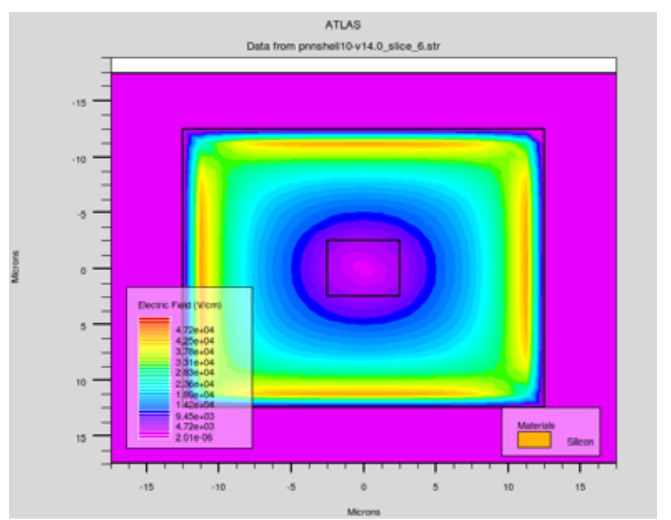

Fig.5 Electric field profile of a detector with an electrode spacing of $10 \mu \mathrm{m}$. The bias voltage is -14 volts. Maximum electric field shown is $5 \times 104 \mathrm{~V} / \mathrm{cm}$.

The detector full depletion voltage can be estimated as:

$$
V_{f d}=\frac{q N_{D} L_{p}^{2}}{2 \varepsilon_{0} \varepsilon}
$$

Here ND is the detector bulk doping concentration. When the electrode spacing is $5 \mu \mathrm{m}$ or $10 \mu \mathrm{m}$, through calculation, we can estimate that the detector full depletion voltage is in the order of a few volts.

\section{Breakdown Consideration}

Even though the absolute values of applied bias voltages are only in the order of 10 volts, due to the nature of device with very small electrode spacing, there could be local high field regions where intrinsic breakdown field for $\mathrm{Si}(\sim 300 \mathrm{KV} / \mathrm{cm})$ may be reached. Such high fields can exist especially near the corners of $p+$ trench, just under the oxide layer. We cut the profile just under the oxide layer (in Z-direction, at $\mathrm{z}=1 \mu \mathrm{m})$ as shown in Fig. 6 ( $\mathrm{Lp}=10 \mu \mathrm{m}$, the bias voltage is -14 volts). From the cutline between two corners of the $\mathrm{p}+$ trench, we can read that the largest value of the electric field is about $37000 \mathrm{~V} / \mathrm{cm}$, which is much below the intrinsic breakdown filed in silicon of $3 \times 10^{5} \mathrm{~V} / \mathrm{cm}$.

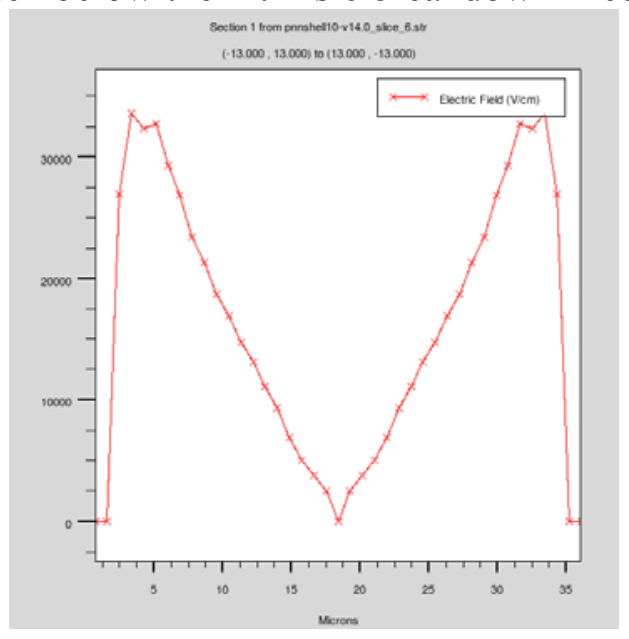

Fig.6 Electric field cutline of a detector with electrode spacing of $10 \mu \mathrm{m}$. The bias voltage is -14 volts.

\section{Summary}

From calculations and full 3D simulations, we conclude that the ultra-fast charge collection time $(\leq$ $100 \mathrm{ps}$ ) can be obtained at a bias voltage of only a few volts (about 10 volts) for small 3D-Trech 
Electrode detectors with electrode spacing less than or equal to $10 \mu \mathrm{m}$. As we simulated in this study, we can get a minimum carrier drift time of about $50 \mathrm{ps}$ in most part of the detector when the detector electrode spacing Lp is $5 \mu \mathrm{m}$, and a minimum carrier drift time of about $100 \mathrm{ps}$ in most part of the detector when $\mathrm{Lp}$ is $10 \mu \mathrm{m}$. The full depletion voltages for these types of detectors are small, less than 10 volts.

Furthermore, because of the nature of the symmetry structure in the all column electrode type 3D detectors, a saddle potential distribution exists in the geometry center of the detector regardless of the detector bias voltage. In the 3D-Trech Electrode detector, the electric potential profile possesses no more saddle points.

Also, we investigated the possible breakdown situation in this type of detectors, and found that at voltages to reach the carrier drift velocity in most part of the detector, the highest electric field in the detector is still much below the value of the Si intrinsic breakdown field. We note here that those type of 3D detectors are intrinsically rad-hard due to their very small electrode spacing.

\section{References}

[1] E. Heijne, "Muon flux measurement with silicon detectors in the CERN neutrino beams," in Proc. CERN-83-06, 1983, p. 18.

[2] K. Borer, A. G. Clark, R. Engelmann, O. Gildemeister, C. Gössling, E. H. M. Heijne, P. Jarron, B. Lisowski, T. Pal, M. A. Parker, and N. Redaelli, "Construction and performance of a 1m silicon detector in UA2," Nucl. Instrum. Methods Phys. Res. A, Accel. Spectrom. Detect. Assoc. Equip., pp. 548-557, 1987.

[3] R. Sonnenblick et al., "Electrostatic simulations for the design of silicon strip detectors and front-end electronics," Nucl. Instrum. Methods Phys. Res. A, Accel. Spectrom. Detect. Assoc. Equip., vol. A 310, p. 189, 1991.

[4] S. I. Parker, C. J. Kenney, and J. Segal, "3D-A proposed new architecture for solid-state radiation detectors," Nucl. Instrum. Methods Phys. Res. A, Accel. Spectrom. Detect. Assoc. Equip., vol. A 395, pp. 328-343, 1997.

[5] C. Da Vià, E. Bolle, K. Einsweiler, M. Garcia-Sciveres, J. Hasi, C. Kenney, V. Linhart, S. Parker, S. Pospisil, O. Rohne, T. Slavicek, S. Watts, and N. Wermes, "3D active edge silicon sensors with different electrode configurations: Radiation hardness and noise performance," Nucl. Instrum. Methods Phys. Res. A, Accel. Spectrom. Detect. Assoc. Equip., vol. A 604, pp. 505-511, 2009.

[6] Sherwood Parker, Angela Kok, Christopher Kenney, Pierre Jarron, Jasmine Hasi, Matthieu Despeisse, Cinzia Da Via, and Giovanni Anelli, "Increased Speed: 3D Silicon Sensors; Fast Current Amplifiers," IEEE TRANSACTIONS ON NUCLEAR SCIENCE, VOL. 58, NO. 2, APRIL 2011.

[7] G. Anelli, F. Faccio, S. Florian, and P. Jarron, "Noise characterization of a $0.25 \mathrm{~m}$ CMOS technology for the LHC experiments," Nucl. Instrum. Methods Phys. Res. A, Accel. Spectrom. Detect. Assoc. Equip., vol. A 457, p. 361, 2001.

[8] G. Anelli et al., "A high-speed low-noise transimpedance amplifier in a $0.25 \mathrm{~m}$ CMOS technology," Nucl. Instrum. Methods Phys. Res. A, Accel. Spectrom. Detect. Assoc. Equip., vol. A 512, p. 117, 2003.

[9] Zheng Li, "Full 3D Simulations on The New BNL ICDA(3D-Trench Electrode) Detectors", 16th Workshop of CERN RD50 - Radiation hard semiconductor devices for very high luminosity colliders, Barcelona, Spain, May 31 to June 2, 2010

[10]Z. Li, New BNL 3D-Trench electrode Si detectors for radiation hard detectors for sLHC and for X-ray applications, Nuclear Instruments and Methods in Physics Research A 658 (2011) 90-97. 
[11]A. Montalbano, D. Bassignana, Z. Li, et al., A systematic study of BNL's 3D-Trench Electrode detectors, Nuclear Instruments and Methods in Physics Research A 765 (2014) 23-28.

[12]J. Chen, H. Ding, Z. Li, et al., 3D simulations of device performance for 3D-Trench electrode detector, Nuclear Instruments and Methods in Physics Research A 796 (2015) 34-37.

[13]H. Ding, J. Chen, Z. Li, et al., Modeling and simulation of charge collection properties for 3D-trench electrode detector, Nuclear Instruments and Methods in Physics Research A 796 (2015) 29-33.

[14]G. F. Dalla Betta, dFondazione Bruno Kessler (FBK), Trento, Italy, private conversions (2014). [15]Atlas User's Manual—device simulation software, Silvaco International. 